authoritative leaders in the field of FTD, is well-written, well organized, and very accessible with numerous tables, illustrations, figures, and case studies. Its chapter on psychological interventions in FTD may be a first, and leaves us wanting more. Both clinically and scientifically, it is a recommended and welcome addition.

\section{RECENT AND RELEVANT}

doi: $10.1017 /$ S1355617708081320

Progress in Neurotherapeutics and Neuropsychopharmacology. Volume 3. Jeffrey L. Cummings (Ed.). 2008. New York: Cambridge University Press, 297 pp., \$180.00 (HB)

Stahl's Essential Psychopharmacology: Neuroscientific Basis and Practical Applications, Third Edition, by Stephen M. Stahl. 2008. New York: Cambridge University Press, 1117 pp., \$175.00 (HB); \$85.00 (PB)

Antipsychotics and Mood Stabilizers: Stahl's Essential Psychopharmacology, Third Edition, by Stephen M. Stahl. 2008. New York: Cambridge University Press, 232 pp., \$125.00 (HB); \$50.00 (PB)

The Normal Personality: A New Way of Thinking About People, by Steven Reiss. 2008. New York: Cambridge University Press, 212 pp., \$26.00 (HB)

Patient-Based Approaches to Cognitive Neuroscience, Second Edition, Martha Farah and Todd E. Feinberg (Eds.). 2006. Cambridge, MA: The MIT Press, 494 pp., \$55.00 (PB)

\section{REFERENCE}

Neary, D., Snowden, J.S., Gustafson, L., Passant, U., Stuss, D., Black, S., Freedman, M., Kertesz, A., Robert, P.H., Albert, M., Boone, K., Miller, B.L., Cummings, J., \& Benson, D.F. (1998). Frontotemporal lobar degeneration. Neurology, 51, 1546-1554.

Study Guide to Neuropsychiatry and Clinical Neurosciences: A Companion to the American Psychiatric Publishing Textbook of Neuropsychiatry and Clinical Neurosciences, Fourth Edition, by James A. Bourgeois, Narriman C. Shahrokh, Robert E. Hales, Stuart C. Yudofsky. 2006. Arlington, VA: American Psychiatric Publishing, 226 pp., \$29.95, (PB)

Prospective Memory: An Overview and Synthesis of an Emerging Field, by Mark A. McDaniel and Gilles O. Einstein. 2007. Los Angeles: Sage Publications, 264 pp., \$49.95 (PB)

From Molecule to Metaphor: A Neural Theory of Language, by Jerome A. Feldman. 2006. Cambridge, MA: The MIT Press, 357 pp., \$36.00 (HB)

Neurobiology: From Molecular Basis to Disease, Robert Meyers (Ed.). 2008. Weinheim, Germany: WileyVCH, 836 pp., \$400.00 (HB) 